\title{
RENDIMIENTO DE HÍBRIDOS DE MAÍZ BAJO DIFERENTES COMBINACIONES DE SEMILLA ANDROESTERIL Y FÉRTIL EN MÉXICO ${ }^{1}$
}

\author{
Alejandro Espinosa-Calderón ${ }^{2}$, Margarita Tadeo-Robledo ${ }^{3}$, Mauro Sierra-Macías ${ }^{4}$, \\ Antonio Turrent-Fernández ${ }^{2}$, Roberto Valdivia-Bernal ${ }^{5}$,Benjamín Zamudio-González $z^{2}$
}

\section{RESUMEN}

Rendimiento de híbridos de maíz bajo diferentes combinaciones de semilla androesteril y fértil en México. El objetivo de este trabajo fue determinar la capacidad productiva de los híbridos comerciales H-48 y Puma 1076 en diferentes mezclas de semilla androesteril y fértil: 1) $100 \%$ semilla androesteril; 2) $90 \%$ semilla androesteril más $10 \%$ semilla fértil; 3) $80 \%$ semilla androesteril más $20 \%$ semilla fértil; 4) $60 \%$ semilla androesteril más $40 \%$ semilla fértil; 5) $40 \%$ semilla androesteril más $60 \%$ semilla fértil; 6) 100 $\%$ semilla fértil. Estas combinaciones fueron evaluadas en el ciclo primavera-verano, desde junio a noviembre, durante los años 2001, 2002 y 2003, en el Campo Experimental Valle de México, a una altitud de $2.250 \mathrm{~m}$. Los experimentos se manejaron con densidad de población de 60.000 plantas/ha. Se utilizó un diseño experimental de bloques completos al azar, y se analizó como factorial. Los factores de variación fueron años, genotipos, combinaciones de semilla androesteril y fértil, así como las interacciones. El tratamiento $90 \%$ semilla androesteril $+10 \%$ semilla fértil tuvo el rendimiento más elevado $(8.738 \mathrm{~kg} / \mathrm{ha})$, superó significativamente al testigo $100 \%$ fértil $(7.488 \mathrm{~kg} / \mathrm{ha})$. La media de los híbridos con esterilidad masculina fue estadísticamente superior en rendimiento de grano con respecto a la versión fértil, lo que representa una ventaja adicional, además de la facilidad para mantener la calidad genética y disminuir los costos en la producción de semilla híbrida de maíz.

Palabras clave: Zea mays, androesterilidad, desespigamiento, producción de semillas, productividad.

\begin{abstract}
Yield of maize hybrids under andro - sterile and fertile seed combinations in Mexico. The objective of this work was to determine the productive capacity of the commercial hybrids H-48 and Puma 1076 in different mixtures from andro-sterile and fertile seed: 1) $100 \%$ andro-sterile seed; 2) $90 \%$ andro-sterile seed plus $10 \%$ fertile seed; 3) 80 $\%$ andro-sterile seed, plus $20 \%$ fertile seed; 4$) .60 \%$ androsterile seed plus $40 \%$ fertile seed; 5) $40 \%$ andro-sterile seed plus 60 fertile seed; and 6) $100 \%$ fertile seed. These combinations were evaluated during the spring-summer seasons, from June to November, during years 2001, 2002 and 2003, in the Experimental Station of Valley of Mexico, located at 2250 masl. The experiments were managed with a density of 60,000 plants/ha. A randomized complete block design was used and analyzed as a factorial. The sources of variation were years, genotypes, combinations of andro-sterile and fertile seed, as well as the interactions. Treatment $90 \%$ androsterile seed $+10 \%$ fertile seed had the highest yield $(8738$ $\mathrm{kg} / \mathrm{ha}$ ), significantly higher than the fertile control $100 \%$ fertile seed, which produced $7488 \mathrm{~kg} / \mathrm{ha}$. The mean of the hybrids with male sterility was superior statistically in grain yield with respect to the fertile version, which represents an additional advantage, in addition to the easiness to maintain the genetic quality and to reduce the costs of the production of hybrid maize seed.
\end{abstract}

Key words: Zea mays, andro-sterility, dettasseling, seed production, productivity.

\footnotetext{
1 Recibido: 21 de octubre, 2008. Aceptado: 16 de noviembre, 2009. Este artículo corresponde al Proyecto de Investigación PAPIIT IN205908.

2 Campo Experimental Valle de México, Instituto Nacional de Investigaciones Forestales, Agrícolas y Pecuarias (INIFAP), km 18.5 Carr. Los Reyes - Lechería, C. P. 56230, Chapingo, México. Teléfono: (595) 9542877 ext. 111, fax: (595) 9546528 . espinoale@yahoo.com.mx; aturrent37@yahoo.com.mx; bzamudiog@yahoo.com.mx

3 Facultad de Estudios Superiores Cuautitlán (FESC)-Universidad Nacional Autónoma de México (UNAM), México. km. 2.5 Carr. CuautitlánTeoloyucan, C.P. 54700. Apdo. Postal 25, Cuautitlán, Edo. de México. teléfono y fax (55) 562319 71. tadeorobledo@yahoo.com

4 Campo Experimental Cotaxtla, Instituto Nacional de Investigaciones Forestales, Agrícolas y Pecuarias (INIFAP), México. mauro_s55@ hotmail.com

5 Universidad Autónoma de Nayarit. beto49_2000@yahoo.com.mx
} 


\section{INTRODUCCIÓN}

En la producción de semilla híbrida de maíz se requiere efectuar adecuadamente el desespigamiento, también llamado despanojamiento en algunos países, para mantener la calidad genética del material de maíz que se obtiene; la eliminación de espigas en forma manual es cara, se requieren de 24 a 50 jornales/ha, que representan un fuerte impacto económico en los costos de producción, de 184 dólares hasta 380 dólares por hectárea, dependiendo de la uniformidad del progenitor femenino, presencia de hijos, facilidad para retirar la espiga (Jugenheimer 1990, Tadeo et al. 2003, Martínez et al. 2005). Una alternativa para facilitar la producción de semilla, así como elevar el nivel de adopción de híbridos de maíz en los Valles Altos, podría ser el uso de tipos y fuentes de androesterilidad génico-citoplásmica, ya que se emplean líneas androestériles como progenitores femeninos, con lo cual se reduce el desespigamiento (Stamp et al. 2000, Martínez et al. 2005).

Desde 1992, investigadores del Instituto Nacional de Investigaciones Forestales, Agrícolas y Pecuarias (INIFAP) y la Universidad Nacional Autónoma de México (UNAM), trabajan con fuentes de esterilidad masculina y su incorporación a los progenitores de híbridos (Tadeo et al. 1997, Tadeo et al. 2003). La androesterilidad, dejó de utilizarse por las empresas de semillas después de los problemas graves de la epifitia en 1970, causada por el uso generalizado de la fuente de esterilidad cms-T, que presentó susceptibilidad a la enfermedad del tizón foliar causada por el hongo Helminthosporium maydis raza T, que ocasionó una epifitia en 90 \% del maíz con cms-T en la faja maicera de los Estados Unidos.

A fines de los 70's y comienzo de los 80's, se desarrollaron nuevos tipos y fuentes de esterilidad masculina (principalmente los tipos C y S), retomándose el uso del esquema de androesterilidad. Actualmente los tipos $\mathrm{C}$ y S están siendo usados en alrededor de un 20 al $30 \%$ del área de producción de semilla en E.U. (Liu et al. 2002, Beck y Torres 2005), tratando de diversificar dentro de cada tipo las fuentes de esterilidad para no depender de una sola y evitar la incidencia de enfermedades (Partas 1997, Liu et al. 2002, Weingartner et al. 2002).

Recientemente en el INIFAP y la UNAM se han desarrollado híbridos con androesterilidad. Para el uso comercial de la semilla obtenida con androesterilidad se emplean dos maneras, para asegurar que cuando se entrega la semilla al agricultor para su siembra comercial, las parcelas de los productores posean plantas fértiles y/o combinación de plantas fértiles y androestériles que aseguren polinización, fecundación y por consecuencia producción de grano. En una de maneras se utiliza un progenitor masculino en la producción de semilla del híbrido, con capacidad restauradora de la fertilidad masculina, lo que significa que restituye la fertilidad masculina (producción de polen) para polinizar a la cruza simple o línea androestéril (dependiendo si se esta incrementando una cruza simple o híbrido trilíneal), este progenitor macho, suprime la androestérilidad y la cruza que se obtiene expresa la fertilidad en la generación siguiente, presentándose en terrenos de los productores el $100 \%$ de las plantas fértiles, el caso más claro de este tipo, es como ocurre en sorgo, donde toda la semilla híbrida se incrementa de esta manera; por lo cual la semilla que adquieren y siembran en sus campos los agricultores, generan plantas completamente fértiles. En la otra manera se emplea la mezcla de proporciones de semilla del híbrido obtenida de hembras androestériles, que mantienen su característica de androestérilidad, que no producen polen, esta semilla se mezcla con una proporción de semilla del mismo híbrido, pero obtenido con progenitores fértiles, que producen polen y en cuya hembra se aplicó desespigue, de esta manera en los campos de los agricultores, se presentarán plantas con esterilidad masculina ( $\sin$ producir polen) y otras con fertilidad normal, las cuales polinizarán al resto de las plantas (Airy et al. 1987, Tadeo et al. 2003, Espinosa et al. 2003a).

La mezcla de la semilla de ambas versiones, debe ser en una proporción adecuada para asegurar la polinización y el rendimiento de grano; esta es la estrategia adoptada actualmente en la mayoría de los materiales de México y otros países por las empresas de semillas que usan la esterilidad masculina (Tadeo et al. 2001, Espinosa et al. 2003b).

Dado que se cuenta con las versiones androestériles y androfértiles de híbridos de INIFAP y la UNAM, si bien se considera que cada híbrido debe ser evaluado en diferentes proporciones de mezcla de semilla androésteril y fértil, en una primera fase se eligió un híbrido de cada una de estas instituciones, con características similares en el ciclo vegetativo. El objetivo de este trabajo fue medir la capacidad productiva de los híbridos comerciales H-48 y Puma 1076 en diferentes proporciones de semilla androésteril y fértil. 


\section{MATERIALES Y MÉTODOS}

Durante el ciclo primavera - verano 2000, se incrementó en el Campo Experimental Valle de México (CEVAMEX), la semilla del híbrido trilíneal H-48 en su versión androestéril, con base en la cruza simple hembra androestéril, y la semilla en la versión de fertilidad normal, y se utilizó la cruza simple fértil. En forma similar en el Rancho Almaraz, de la Facultad de Estudios Superiores Cuautitlán, de la Universidad Nacional Autónoma de México (UNAM), se incrementó semilla del híbrido de maíz Puma 1076, en su versión androestéril y fértil. Para ambos híbridos se mezclaron semillas en varias proporciones; androesteril y fértil: 1) $100 \%$ semilla androesteril; 2) $90 \%$ semilla androesteril más $10 \%$ semilla fértil; 3) $80 \%$ semilla androesteril más $20 \%$ semilla fértil; 4) $60 \%$ semilla androesteril más $40 \%$ semilla fértil; 5) $40 \%$ semilla androesteril más $60 \%$ semilla fértil; 6) $100 \%$ semilla fértil.

Los dos híbridos fueron evaluados durante los ciclos Primavera - Verano 2001, 2002 y 2003 en el Campo Experimental Valle de México (CEVAMEX), en parcelas experimentales de cuatro surcos de cinco metros de largo y $0,80 \mathrm{~m}$ entre surcos, para utilizar dos centrales como parcela útil. La fecha de siembra fue el 29 de mayo de 2001, 6 de julio de 2002, 20 de mayo de 2003. En los tres años se dio un riego de siembra y las plantas se desarrollaron bajo condiciones de temporal. La densidad de población fue de 60.000 plantas por hectárea, y la fertilización de 150-7030. En los tres años se utilizó el diseño de bloques completos al azar, con cuatro repeticiones y arreglo factorial, los factores de variación: años (3), genotipos (2), combinaciones de semilla androesteril y fértil
(6), así como las interacciones entre los factores en estudio. Las variables analizadas fueron: rendimiento, diámetro, longitud, número de hileras por mazorca, granos por hilera, peso de 200 semillas, estos datos se tomaron de cinco mazorcas por parcela. La separación de medias se hizo con la prueba de Tukey al 0,05 de probabilidad.

\section{RESULTADOS Y DISCUSIÓN}

Para la variable rendimiento, los resultados del análisis de varianza considerando años, genotipos, combinación de porcentajes de semilla androestéril/ fértil y las interacciones genotipos por combinación de porcentajes de semillas, año por genotipo, y por combinación de porcentajes, definieron que el coeficiente de variación fue de $17,1 \%$ y la media general de rendimiento de $8,274 \mathrm{~kg} / \mathrm{ha}$. Se detectó diferencia altamente significativa para los factores de variación años, genotipos, repeticiones, y la interacción años x genotipos, así como diferencias significativas $(0,05$ de probabilidad) para el factor combinación de porcentajes de semilla androesteril/fértil. El coeficiente de variación más alto se obtuvo con el rendimiento $(17,1 \%)$.

En el ciclo primavera - verano 2002 se obtuvo una media de producción de $9.647 \mathrm{~kg} / \mathrm{ha}$, diferente estadísticamente a la media de producción obtenida en el ciclo primavera - verano 2001 y primavera - verano 2003, en los cuales el rendimiento medio fue $7.725 \mathrm{~kg} / \mathrm{ha} \mathrm{y}$ $7.451 \mathrm{~kg} / \mathrm{ha}$, respectivamente (Cuadro 1). En las otras variables evaluadas con respecto a los tres años, se presentó un solo grupo de significancia. Lo anterior señala que para cada uno de los años si bien hubo diferencia

Cuadro 1. Medias de seis variables medidas en las combinaciones androestériles y fértiles de semilla de H-48 y Puma 1076. Campo Experimental Valle de México, México. Ciclos primavera - verano 2001 a 2003.

\begin{tabular}{ccccccc}
\hline \multicolumn{1}{c}{ Año } & Rend. kg/ha & DM $(\mathbf{c m})$ & NH & GH & LM $(\mathbf{c m})$ & PS (g) \\
\hline PV 2001 & $7725 \mathrm{~b} *$ & 4,7 & 15 & 27 & 14,4 & 36,8 \\
PV 2002 & $9647 \mathrm{a}$ & 4,7 & 15 & 26 & 14,3 & 37,8 \\
PV 2003 & $7451 \mathrm{~b}$ & 4,7 & 15 & 26 & 14,3 & 38,0 \\
DSH (0,05) & 686 & 0,09 & 0,4 & 1,2 & 1,1 & 2,7 \\
\hline
\end{tabular}

* Medias con letras iguales no son estadísticamente diferentes (Tukey 0,05).

DM: Diámetro de mazorca; NH: Número de hileras; GH: Granos por hilera; LM: Longitud de mazorca; PS: Peso de 200 semillas. 
en producción general, debida probablemente a las condiciones de precipitación y otros factores ambientales, lo que influyó en el rendimiento, en cambio en el promedio de las versiones androestériles y fértiles de los híbridos evaluados, no hubo efecto significativo.

En promedio de los tres años de evaluación y las combinaciones de porcentajes de semilla androestéril/fértil, el híbrido de maíz Puma 1076 expresó una producción media de $8.638 \mathrm{~kg} / \mathrm{ha}$, que superó al híbrido de INIFAP denominado H-48, el cual rindió $7.911 \mathrm{~kg} / \mathrm{ha}$ (Cuadro 2), esta diferencia se debe a la conformación genética de los materiales (Espinosa et al. 2003b, Tadeo et al. 2004), lo que se observa en las diferencias de las variables evaluadas, en donde Puma 1076 presentó valores superiores en diámetro de mazorca, granos por hilera, longitud de mazorca, peso de 200 semillas, con respecto al H-48 (Cuadro 2).

El rendimiento medio de los híbridos Puma 1076 y H-48, representan niveles de producción aceptables, si se consideran los registros en otros experimentos y reportes de cada material, los valores obtenidos se ubican dentro de lo que podría esperarse (Tadeo et al. 2003, Espinosa et al. (2003b), ambos materiales se utilizan en forma comercial.

Con respecto a los tratamientos de combinaciones de porcentajes de semilla androestéril/fértil, el tratamiento de $90 \%$ semilla androestéril $+10 \%$ semilla fértil expresó un rendimiento de $8.738 \mathrm{~kg} / \mathrm{ha}$, siendo el valor más alto, estadísticamente superior al testigo $100 \%$ fértil, el cual produjo $7.488 \mathrm{~kg} / \mathrm{ha}$, si bien las causas del aumento en rendimiento de semilla de versiones con androesterilidad sobre las fértiles como en este caso, no han sido dilucidadas, Urs et al. (2002) mencionan que se desconoce hasta que punto la esterilidad del polen per se contribuye a las diferencias en el rendimiento de grano entre materiales. Se ha tratado de explicar que en la formación de polen fértil se requiere una demanda poderosa de nutrimentos tales como nitrógeno, de modo que su abastecimiento a los órganos femeninos se reduce y ocasiona disminución del rendimiento potencial de semilla (Martínez et al. 2005).

Otras combinaciones de semilla androestéril/fértil como $90 \%, 80 \%, 100 \%$ y $60 \%$ mostraron diferencias de 16,$6 ; 15,8 ; 14,5 \%$ y $11,6 \%$ con respecto a la versión 100 por ciento fértil, lo que coincide con resultados de otros trabajos (Fleming et al. 1960, Simeonov 1995, Stamp et al. 2000, Urs et al. 2002, Martínez et al. 2005), cabe aclarar que el tratamiento $100 \%$ androestéril no es aplicable en forma práctica, porque se requiere una fracción de plantas fértiles para que ocurra la polinización y fecundación del resto de plantas, en parte también ésto podría explicar parcialmente la buena respuesta del tratamiento $90 \%$ semilla androestéril $+10 \%$ semilla fértil, ya que el tratamiento $100 \%$ androestéril podría haber tenido alguna dificultad de polinización y fecundación oportuna a diferencia del tratamiento donde el $10 \%$ es fértil (Cuadro 3).

La diferencia estadística media de las combinaciones con semilla androestéril en porcentajes de $90 \%, 80 \%$ y $60 \%$ indican que la androesterilidad otorga alguna ventaja en el híbrido final, contrario a lo reportado para las cruzas simples progenitoras de los mismos híbridos (Espinosa et al. 2003b), pero en concordancia con trabajos previamente reportados

Cuadro 2. Medias de las seis variables de los híbridos Puma 1076 y H-48 evaluados durante tres años bajo diferentes combinaciones androesteriles y fértiles de semilla. Campo Experimental Valle de México, México. Ciclos primavera - verano 2001 a 2003.

\begin{tabular}{lcccccc}
\hline \multicolumn{1}{c}{ Híbrido } & $\begin{array}{c}\text { Rend. } \\
\text { kg/ha }\end{array}$ & $\begin{array}{c}\text { D.M. } \\
(\mathbf{c m})\end{array}$ & N.H. & G.H. & $\begin{array}{c}\text { L.M. } \\
(\mathbf{c m})\end{array}$ & $\begin{array}{c}\text { P.S. } \\
(\mathbf{g})\end{array}$ \\
\hline Puma 1076 & $8.638 \mathrm{a} *$ & $4,7 \mathrm{a}$ & $15 \mathrm{a}$ & $28 \mathrm{a}$ & $14,8 \mathrm{a}$ & $40,9 \mathrm{a}$ \\
H 48 & $7.911 \mathrm{~b}$ & $4,6 \mathrm{~b}$ & $15 \mathrm{a}$ & $26 \mathrm{~b}$ & $13,8 \mathrm{~b}$ & $34,1 \mathrm{~b}$ \\
D.S.H. $(0,05)$ & 467 & 0,06 & 0,3 & 0,8 & 0,7 & 1,8 \\
\hline
\end{tabular}

* Medias con letras iguales no son estadísticamente diferentes (Tukey 0,05 )

D.M.: Diámetro de mazorca; N.H.: Número de hileras; G.H.: Granos por hilera; L.M.: Longitud de mazorca; PS: Peso de 200 semillas. 
Cuadro 3. Medias de seis diferentes combinaciones de semilla androestéril y fértil de los híbridos Puma 1076 y H-48 evaluados durante tres años. Campo Experimental Valle de México, México. Ciclos Primavera - Verano 2001 a 2003.

\begin{tabular}{lccccccc}
\hline Combinación AE/Fértil & $\begin{array}{c}\text { Rend. } \\
\text { (kg/ha) }\end{array}$ & $\begin{array}{c}\text { \% Sobre } \\
\mathbf{1 0 0} \% \mathbf{~ F}\end{array}$ & $\begin{array}{c}\text { D.M. } \\
(\mathbf{c m})\end{array}$ & N.H. & G.H. & L.M. & $\begin{array}{c}\text { P.S. } \\
(\mathbf{g})\end{array}$ \\
\hline $90 \% \mathrm{AE}+10 \% \mathrm{~F}$ & $8.738 \mathrm{a}$ & 116,6 & 4,6 & 15 & 26 & 13,9 & $41,2 \mathrm{a}$ \\
$100 \% \mathrm{AE}$ & $8.671 \mathrm{ab}$ & 115,8 & 4,5 & 15 & 27 & 14,3 & $37,9 \mathrm{ab}$ \\
$80 \% \mathrm{AE}+20 \% \mathrm{~F}$ & $8.575 \mathrm{ab}$ & 114,5 & 4,6 & 15 & 27 & 13,8 & $35,0 \mathrm{~b}$ \\
$60 \% \mathrm{AE}+40 \% \mathrm{~F}$ & $8.359 \mathrm{ab}$ & 111,6 & 4,6 & 15 & 27 & 15,7 & $36,2 \mathrm{~b}$ \\
$40 \% \mathrm{AE}+60 \% \mathrm{~F}$ & $7.815 \mathrm{ab}$ & 104,4 & 4,6 & 15 & 26 & 14,0 & $36,0 \mathrm{~b}$ \\
$100 \% \mathrm{~F}$ & $7.488 \mathrm{~b}$ & 100 & 4,7 & 15 & 27 & 14,1 & $38,9 \mathrm{ab}$ \\
D.S.H. $(0,05)$ & 1.185 & & 0,15 & 0,78 & 2,2 & 1,9 & 4,7 \\
\hline
\end{tabular}

* Medias con letras iguales no son estadísticamente diferentes (Tukey 0,05).

D.M.: Diámetro de mazorca; N.H.: Número de hileras; G.H.: Granos por hilera; L.M.: Longitud de mazorca; P.S.: Peso de 200 semillas.

(Fleming et al. 1960, Simeonov 1995, Stamp et al. 2000, Urs et al. 2002).

El híbrido Puma 1076 superó estadísticamente al H-48 en 727 kg/ha. La esterilidad masculina en los híbridos $\mathrm{H}-48 \mathrm{AE}^{8}$ y Puma $1076 \mathrm{AEC}^{8}$ otorga ventaja en rendimiento de grano con respecto a la versión fértil, lo cual es adicional a la facilidad para la producción de semilla al disminuir los costos de producción de semilla de híbridos de maíz por la reducción del desespigue manual.

\section{CONCLUSIONES}

Se determinó estadísticamente, durante los ciclos 2001, 2002 y 2003, que la media de todas las versiones androestériles de H-48 y Puma 1076 expresaron un rendimiento superior a la versión fértil.

La media del híbrido Puma 1076 (8.638 kg/ha), considerando las diferentes combinaciones de androesterilidad y fertilidad a través de los tres años fue estadísticamente superior al híbrido $\mathrm{H}-48$, lo que representa $109,2 \%$.

El tratamiento $90 \%$ semilla androestéril $+10 \%$ semilla fértil $(8.671 \mathrm{~kg} / \mathrm{ha})$, superó estadísticamente al testigo $100 \%$ fértil $(7.488 \mathrm{~kg} / \mathrm{ha})$ y las combinaciones de semilla con $90 \%, 80 \%$ y $60 \%$ semilla androestéril,

\footnotetext{
${ }^{8}$ Androestéril.
}

ISSN: 1021-7444 con la contraparte de semilla fértil, produjeron 16,6 $\%, 15,8 \%, 14,5 \%$ y $11,6 \%$ más con respecto al tratamiento $100 \%$ fértil.

La superioridad en rendimiento por la esterilidad masculina en los híbridos H-48 y Puma 1076, representa una ventaja adicional con respecto a la versión fértil y facilidad para la producción de semilla al disminuir los costos de producción de híbridos de maíz, al no hacer el desespigue.

\section{AGRADECIMIENTO}

Los autores agradecen el apoyo financiero del Programa de Apoyo a Proyectos de Investigación e Innovación Tecnológica (PAPIIT IN205908) de la Universidad Nacional Autónoma de México (UNAM) para la realización de la presente investigación.

\section{LITERATURA CITADA}

Airy, JM; L. A. Tatum; Soreson, JW. 1987. La producción de semillas. Producción de semilla híbrida de maíz y sorgo para grano. Editorial C.E.C.S.A. p. 274-285.

Beck, DL; Torres, JL. 2005. Desespigamiento. In: Ortiz T, C, A. Espinosa C, HS Azpiroz R y S Sahagún C. (Comps.). Producción y tecnología de semillas de maíz INIFAP para los Valles Altos y Zonas de Transición. 
Libro Técnico Núm. 3. Campo Experimental Valle de Toluca, CIRCE, INIFAP. Zinacantepec, México. p. 44-55.

Espinosa, A; Sierra, M; Gómez, N; Reyes, C; Caballero, F; Tadeo, M; Palafox, A; Cano, O; Rodríguez, F; Betanzos, E; Coutiño, B. 2003a. Seed production and andro-sterilite in normal and quality protein maize. In: Books of Abstract: Arnel R. Hallauer International Symposium on Plant Breeding. México City. D.F. México. p. 238-239.

Espinosa, A; Tadeo, M; Lothrop, J; Azpiroz, S; Martínez, R; Pérez, JP; Tut, C; Bonilla, J; Ramírez, AN; Salinas, Y. 2003b. H-48 nuevo híbrido de maíz de temporal para los Valles Altos del centro de México. Agricultura Técnica en México 29(1):85-87.

Fleming, AA; Koselnicky, GM; Browne, EB. 1960. Cytoplasmic effect on agronomic characters in a double cross maize hybrid. Agron. J. 52:112-115.

Jugenheimer, RW. 1990. Maíz, variedades mejoradas, métodos de cultivo y producción de semillas. Ed. Limusa, México. p. 489-502.

Liu Z, S; Peter, O; M. Long; Weingartner, U; Stamp, P; Kaeser, O. 2002. A PCR assay for rapid discrimination of sterile cytoplasm types in maize. Crop Science 42:566-569.

Martínez-Lazaro, C; Mendoza-Onofre, LE; García-de Los Santos, G; Mendoza-Castillo, M del C; MartínezGarza, A. 2005. Producción de semilla híbrida de maíz con líneas androestériles y androestériles isogénicas y su respuesta a la fertilización y densidad de población. Revista Fitotecnia Mexica 28(2):127-133.

Partas, EK. 1997. Male sterility as an efficient method of exploiting heterosis in maize. In: The Genetics an
Exploitation of Heterosis in Crops. An International Symposium. México. p. 244-245.

Stamp, P; Chowchong, S; Menzi, M; Weingartner, U; Kaeser, O. 2000. Increase in the yield of cytoplasmic male sterile maize revisited. Crop Science 40:1586-1587.

Simeonov, N. I. 1995. Efect of male-sterility cytoplasm and mode of restoration on the seed production and yield of the single and modified maize hybrid knezha 530. In: Rasteniev“ dni-Nauki. 32 9-10 y 86-89.

Tadeo, M; Espinosa, A; Martínez, R; Solano, AM; Piña, A. 1997. Use of CIMMYT germplasm to develop maize hybrids at the UNAM. In: The Genetics and Exploitation of Heterosis in Crops. An International Symposium México. p. 240-241.

Tadeo, M; Espinosa, A; Solano, AM; R. Martínez M. 2001. Esterilidad masculina para producir semilla híbrida de maíz. Ciencia y Desarrollo 157: 64-75.

Tadeo, M; Espinosa, A; Solano, AM; Martínez, R. 2003. Androesterilidad en líneas e híbridos de maíz de Valles Altos de México. Agronomía Mesoamericana 14(1): 15-19.

Tadeo, M; Espinosa, A; Martínez, R; Srinivasan, G; Beck, D; Lothrop, J; Torres, JL; Azpiroz, S. 2004. Puma 1075 y Puma 1076 híbridos de maíz de temporal para los Valles Altos de México (2.200 a 2.600 msnm). Revista Fitotecnia Mexicana 27(2):211-212.

Urs, W; Kaeser, O; Long, M; Stamp, P. 2002. Combining cytoplasmic male sterility and xenia increases grain yield of maize hybrids. Crop Science 42:1848-1856.

Weingartner, U; Prest, TJ; Camp, KH; Stamp, P. 2002. The Plus-hybrid system: a method to increase grain yield by combined cytoplasmic male sterility and xenia. Maydica 47:127-134. 